\title{
Exploring visualisation in web information retrieval
}

\section{Anwar Alhenshiri* and James Blustein}

Faculty of Computer Science,

Dalhousie University,

6050 University Avenue, Halifax, NS, B3H1W5, Canada

E-mail: anwar@cs.dal.ca

E-mail: jamie@cs.dal.ca

*Corresponding author

\begin{abstract}
Most conventional web search engines return a small fraction of results per display in textual format. Such renderings require the user to scroll over multiple pages to find relevant results. To enhance the user's ability to identify relevant documents among large sets of results, visualisation techniques are suggested. In addition, integrating the user in the process of query reformulation - by visualising the process itself - can lead to more relevant search results. This paper explores research concerned with visualising the processes of query reformulation and results rendering in web search, and provides research recommendations for further investigations of how best to integrate visualisation into web information retrieval.
\end{abstract}

Keywords: web information retrieval; web IR; information visualisation; query reformulation.

Reference to this paper should be made as follows: Alhenshiri, A. and Blustein, J. (2011) 'Exploring visualisation in web information retrieval', Int. J. Internet Technology and Secured Transactions, Vol. 3, No. 3, pp.320-330.

Biographical notes: Anwar Alhenshiri is a PhD candidate at the Faculty of Computer Science, Dalhousie University. He received his Master's degree in Computer Science from the University of Western Ontario in 2007. He worked at the Daewoo automaker for three years as a System Designer and a as Programmer between 1998 and 2000. He has also worked as a member of the system group at the Faculty of Engineering, Misurata University between 2000 and 2004. He worked as a Lecturer at the Faculty of Information Technology, Misurata University in 2007 and 2008. He is a member of the Institute of Electrical and Electronic Engineers (IEEE). $\mathrm{He}$ has several refereed publications. His main research interests are information retrieval, web search, human-computer interaction and information visualisation.

James Blustein is employed as an Associate Professor in the Faculty of Computer Science and the School of Information Management at Dalhousie University where he heads the Hypertext Augmenting Intelligent Knowledge Use (HAIKU) project. His research motivation is to help people to find and use information. His primary research interests are in hypertext and human-computer interaction.

Copyright (C) 2011 Inderscience Enterprises Ltd. 


\section{Introduction}

People trying to find information 'on' the World Wide Web (WWW) encounter the cognitive impediments necessitated by interacting with search engines. Because users' information needs are necessarily mediated through search engines, users have to split their attention between their goal (of satisfying an information need) and the immediate task (of working with the search engine). Our objective in the research survey we present here is to lead to ways to reduce the impediment.

To interact with traditional search engines, e.g., Google, users enter queries in the form of sequences of words and select from the results (hits) or enter a different query. The process of selecting a new query is known as query reformulation.

Query reformulation in conventional search engines usually requires the user to either choose from an alternative set of queries provided by the engine or to write a query using different keywords from those used in the original query. Web search engines typically suggest alternative requests based on the frequency of answered queries. Such approaches are often problematic since they provide suggestions unrelated to users' actual information need. More than wasting time, such suggestions can distract users' from their real goal. Furthermore, users must read several alternatives to decide which, if any, of the suggestion is useful. Since users have no control over the alternatives, the chance of satisfying the user's need is reduced.

The rendering process (that is, presentation of results) in most conventional search engines presents several search results in the form of links with limited text to indicate the content at the destination of the link. Web search often results in high recall but low precision - meaning that although the most relevant results are often presented to users by search engines, the results are often inappropriately ranked. Users must scroll over multiple web pages to find their intended results (Roberts et al., 2004). However, about $87 \%$ of web search users usually do not look beyond the first three results (Spink et al., 2001; Hoeber and Yang, 2004) before they reformulate their query. When searches are ineffective, users typically modify the search query or switch to a different search tool (Solis-Oba and Alhenshiri, 2007).

This research is an investigation into how visualisation might improve web information retrieval (web IR). Integrating visualisation in web search combines computation and human perception (Vincente and Rasmussen, 1990). Visualisation can be integrated in web IR in three ways. First, visualisation can be applied to web mining. Second, visualisation can benefit query reformulation. Finally and most importantly, visualisation can be involved in the rendering step of web search. The focus of this survey is mainly on the second and third aspects.

We review several visualisation techniques for query reformulation and presentation of search results. We particularly focus on findings that suggest how visualisation could best be used to make searching more effective. The remainder of this article is organised as follows. Section 2 discusses the methodology chosen for collecting and analysing research resources. In Section 3, we review research related to visualisation in web IR. In Section 4 we draw lessons from the findings in Section 3. Section 5 provides recommendations for further research opportunities in visualising the process of web IR. 


\section{Methodology}

We surveyed articles from the IEEE (http:/www.computer.org/portal/web/csdl) and ACM (http://portal.acm.org/dl.cfm) digital libraries. Specifically, we gathered articles associated with the following terms: visualisation of large data sets, information visualisation for text analysis, adaptive visualisation, visualisation of web search results for effective retrieval, visual query formulation, and visualisation for reducing the dimensionality of search results. Our particular interest in this survey was articles about visualisation for the query reformulation and rendering stages of web IR for the reasons outlined in the introduction above.

The research on query reformulation that we analysed involved articles that dealt with visualising query term frequency, dynamic reformulation and visualisation of query terms, and visualising the process of assigning levels of significance to query terms. Research about results presentation included the visualising features of web search results, visualising the organisation of search results, visualising the presentation of result hits, and visual clustering of web results. The following section describes aspects of some of those topics.

\section{Research rationale}

The two main stages of web IR in which visualisation is usually employed are the results presentation and query reformulation. Consequently, the use of visualisation in these stages is usually considered for helping users to

a best use search results

b construct more successful search queries.

Visualisation can help users to perceive relevant results in the list of returned documents, and explore those results in different ways. Visualisation may improve search efficiency and users' satisfaction too. The following sections discuss the use of visualisation in these two stages, making practical recommendations for improvement.

\subsection{Visualising the query reformulation stage}

Most web search engines render ranked search results over multiple web pages. Users usually find it inconvenient to scroll through several pages to find their intended results. Most web search engine users do not usually look beyond the first three results (Spink et al., 2001; Hoeber and Yang, 2009). Consequently, they may either restart the search operation, or reformulate their queries by providing different keywords (Marchionini, 1997). The emphasis on query formulation in the research literature has almost always been on system-controlled approaches which have had limited effects on the relevancy of search results (Miller, 1990; Manning et al., 2008).

The use of query synonyms or profile-content-related terms for constructing alternative requests to the original user query, is usually done without consulting the user, and is based only on term similarities between the original and reformulated 
queries. For example, Havre et al. (2001) introduced a technique that visualises the results of multiple queries generated by the system as alternatives to a user query. Moreover, Suvanaphen and Roberts (2004) designed a search interface that allows users to compare sets of results rendered to multiple queries also generated on behalf of the user. Applying these methods often results in redundant and irrelevant results due to search engines' lack of knowledge about the actual information need of the user (Hoeber and Yang, 2009). Consequently, exploiting visualisation during the process of query reformulation by allowing the user to interact with the search tool is suggested to lead to better expression of the actual user information need. The following sections analyse several studies that looked at adapting the process of query reformulation to the user.

\subsubsection{Visual query expansion}

Hoeber and Yang (2009), motivated by the need for interactive query refinement, suggested a colour histogram that provided useful means for presenting and selecting alternative terms from the top results returned by a conventional search engine. The search process they employed selected those terms based on their similarity to the keywords of the original query as well as their frequency in the top results. Users had the ability to choose from terms in the presented list to add to the original query, or alternatively remove existing terms for the purpose of constructing a different query. The choice of terms for the reformulated query reflected immediately on the results' portion of the display. Further enhancements to the query could be continuously applied. However, the approach suffered from 'visual noise' because of the extensive use of colour variances for keywords.

Similarly, Kawano (2000) designed a search engine that extended the original user query to enhance the relevancy of the final search results. Extending the original query was done by reformulating the query using keywords from the database of web documents gathered by a companion web crawler. Using associative rules, the additional keywords were selected from metadata and other parts of the preserved HTML documents. The resulting reformulated query was presented visually to the user as a multi-dimensional graph. The interactive graphical interface in this approach allowed its users to see the keywords derived from web documents with their associated weights. Users could interactively select keywords for their queries using Boolean operators (AND/OR/NOT). User satisfaction was recorded with this method. A similar idea can be seen implemented in the search engine Quintura (http://www.quintura.com/).

\subsubsection{Visual weight assignment to query terms}

In web IR, the contribution of each term in a multi-term query to the overall relevance of the final results is usually overlooked and not thoroughly investigated. Bonnel et al. (2006) argued that permitting the user to assign ranks to query terms may provide more satisfactory results. Hence, Grewal et al. (2000) introduced a visualisation technique called 'result wheel' (or R-wheel for short). The purpose of this mechanism was to allow users to indicate the relative significance of each query term by shading the term's corresponding segment on the graphically presented wheel (as a portion of the whole). Testing showed that the R-wheel approach permitted users to correctly rank the returned results by assigning term relevance to the query. 


\subsection{Visualising the results presentation stage}

One important problem with current rendering techniques in web search is the inability to sufficiently and effectively exploit the computer display. Currently, users have to read a great deal of text on the screen and are often expected to scroll over multiple pages to find documents they seek (Roberts et al., 2004). With the advancements achieved in designing effective search tools, there is almost always a good indication that the intended results by the user are among the located set of matches (i.e., the level of recall is high). However, the large numbers of matching documents and the current design of textual rendering make it hard for users to perceive relevant results. Consequently, visualisation is suggested to improve users' performance by harnessing their innate abilities to detect relevant matches (Card et al., 1999). Maximising the display capabilities as well as visually expressing more features of web search results are factors anticipated to have a noticeable impact on relevancy.

\subsubsection{Visual objects provide better perception}

Mukherjea and Hara (1999) investigated a spatial hypertext-based technique that uses the scatter plot and the bird's eye layouts for a tabular visualisation of search results. In the tabular bird's eye layout, the size, colour, and position properties represented the keyword match, the knowledge domain, and the term-based rank of the presented documents respectively. The scatter plot visualisation was particularly intended for rendering search results as thumbnails of the corresponding pages. The interface used an underlying search engine to derive web search results. Although user satisfaction was recorded, the approach was limited to a certain number of results. The reported version also suffered from inefficiency issues related to response delays.

Utilising clustering along with visualisation for rendering web search results was investigated by Bonnel et al. (2000). They used a self-organising map (SOM) to cluster results for rendering. The goal was to obtain various search topics from the collection of returned results. In this approach, each topic is labelled with the most frequent terms in its related documents. The clusters were presented in a mixed-mode visual metaphor. A '3D city' metaphor was implemented to help users to recognise different clusters based on colour differences. The rank of the rendered documents was distinguished by the height of each building on the display. Additionally, the interconnectedness within each cluster was represented by the position of buildings on the city metaphor layout. A grid of the ground distribution of clusters was also provided to the user in a 2D representation. This approach was shown to be effective compared to textual rendering techniques but only when used with a small number of search results.

Joho and Jose (2006) investigated the effectiveness of four presentation layouts for search results namely: text presentation, text accompanied with three bolded top ranked sentences (TRS) within a page, text with thumbnails of the pages, and text with TRS and thumbnails. The study showed that users found the presentation of text summaries accompanied with thumbnails and the TRS to be the most effective. Finally, Teevan et al. (2009) evaluated the relative effectiveness of visual snippets, text, and thumbnail presentations. Visual snippets consisted of thumbnails augmented with selected parts of the text on the web page. Visual snippets permitted users to complete their search and research tasks faster and more effectively than any of the other presentations. 


\subsubsection{Satisfying the demand for more interactivity}

Motivated by the need for more focus on assisting users to interactively explore large data sets, Zaveskey et al. (2008) presented an algorithm that provided shorter browsing time, intuitive understanding of results, and non-linear exploration. The investigated 'Visual Islands' presented maximised feature contrast and grouped together similar concepts (clustering) by abstraction. The three main design aspects were coherence and diversity, intuitive display, and engaged guided browsing. To evaluate coherence and diversity, speed and accuracy were measured. Measuring the 'intuitive-ness' of the display as well as the engaged guided browsing method were done using a user preference evaluation test. The Visual Islands were shown to be more coherent, intuitive, and engaging than other methods involved in the comparison. Several questions were left for further work, such as the choice of data clustering algorithm based on data statistics.

Similarly, Cellary et al. (2004) examined varied characteristics of web search results. They embedded interactivity in the visual presentation to permit users to select the type of visualisation and the search attributes. In the 'Periscope' project, the 3D metaphor consisted of multiple levels corresponding to different categorisation aspects of the search results such as the source server of the web documents. Glyphs were laid-out on multiple planes and organised so that each plane represented a dimension to be visualised. A dimension described a common property held by all glyphs in the plane such as size range or creation date range. Users could navigate through the scene and explore features of the search results such as size and source server. Although a wide range of web document features was visualised in addition to the implemented categorisation of the rendered results, the display conveyed noise and clutter. Implications have been made such that noise in the case of non-professional web search users degrades the effectiveness of visualisation (Abrams and Law, 2002; Shidara and Richmond, 2005). In addition, familiarity of the users with textual presentations was noticeably ignored in this approach. As a result, users might find it difficult to adapt to the Periscope interface (Alonso and Baeza-Yates, 2003).

\subsubsection{Presenting the big picture with less computational demand}

Although techniques that use visual presentations as their standard rendering methods, such as the search engine Kartoo (http://www.kartoo.com/), provide additional features over purely text-based approaches, these techniques have not yet been widely adopted by web search users (Kunz and Botsch, 2002). The familiarity with current web search engines and their text-based rendering seems to be causing users to avoid visualisation-driven tools as indicated by Alonso and Baeza-Yates (2003). Therefore, they introduced a visualisation method for web search results that, although text-based, provided better than average rendering. The design provided two text views of the results rendered to the user which are the 'strip view' and the 'thumbnail view'. Many documents were presented at once. The user could hover over the text strips to see the content of the corresponding documents in a separate view (thumbnail view) on a different part of the display.

The thumbnail view provided additional features over the strip view. For example, users could see the query keywords in the document content that was presented in small font so that documents could be compared instantly. Even though this technique did not permit representing many features of web search results such as document size, 
corresponding knowledge domain, and ranking properties, the cost was significantly less than other mechanisms. However, further improvements to results rendering based on text only are hard to envision because of the relatively limited characteristics of text when compared to visual components such as $3 \mathrm{D}$ objects and images.

\subsubsection{Visualisation for effective comparison and decision making}

Havre et al. (2001) introduced 'Sparkler', a technique that generates multiple alternate queries using components (keywords and phrases) in the original user query and visualises the results of all queries. The interface also shows the contribution of each query alternative/component to the overall relevance of each document in the result set. The usability test showed that users preferred Sparkler to the row presentation because they could easily perceive the differences between the initial query and its alternatives. Query components/alternatives were exploited to provide effective comparisons of the search results. However, users were not effectively incorporated in the query reformulation process.

Suvanaphen and Roberts (2004) designed a search interface that allowed users to submit several queries and compare sets of results rendered to those queries. The objective was to permit users to observe similarities and differences among the result sets, reduce the cognitive effort that would result from switching from one result set to another, and enable users to browse more effectively. Although this technique was effective for comparing search results, the evaluation revealed scalability problems and difficulties with using the interface.

\section{Discussion}

To date, research in the field of web information visualisation has investigated several aspects. Two stages of the web IR process have been the focus of concentration with the intent of improving search success (measured by effectiveness, user satisfaction, and to a lesser extent by efficiency) by applying visualisation techniques. Those two stages are query reformulation and the presentation of results (also known as rendering). Regarding improving relevancy in web search results by visualising the query reformulation stage, research has focused on permitting users to interactively participate in query reformulation. In most conventional search engines, a user submits a query and receives results. When no relevant results are presented to the user, that user must either submit an entirely different query, reformulate the current request based on their background knowledge of the search-topic-related keywords, or give up. When using visual techniques, the user is presented with ways to reformulate their queries including providing alternative terms inferred from different sources or assigning priority levels to terms in the original query. These methods have been investigated and shown to allow users to identify potentially more effective queries.

The second stage of the web IR process, also emphasised with respect to utilising visualisation, is the results' presentation stage. Final results of current conventional search engines are presented in textual format. To find their intended documents, users are often expected to scan a great deal of text, scroll over multiple pages, and perhaps resubmit different search queries. Therefore, to overcome the tediousness and time consumption in attempting to find relevant results, visualisation has been applied. 
Rendering is modified so that the display permits users to see more results and document features, to navigate through the results easily, and to perceive the significance of each matching document readily and quickly. Several techniques have been investigated such as tabular presentations, city metaphors, strip and thumbnail views, and periscopes.

All of those techniques are reported to have improved user navigation, search performance (effectiveness) and satisfaction over conventional techniques. Because of those improvements, users perceive that the results of their searches are more relevant than the same results presented in other formats. However, technical obstacles and processing delays because of using visual objects, especially in the case of the web, remain in the path of providing efficient visualisation tools for large collections of results.

It is clear that for many users visualisation will be a great boon however. Alhenshiri et al. (2010a) investigated the effectiveness of visualisation in improving information gathering tasks by creating VSE (shown in Figure 1), a search engine incorporating visualisation. In their evaluation of VSE, users found greater satisfaction, plus faster and more efficient searches than conventional search engines. Compared to Google, the VSE permitted its users to gather information required in the task more efficiently. Moreover, users of VSE submitted fewer search queries to finish the tasks, required opening and viewing fewer web pages, and found more relevant results. Clearly, there is great potential for visualisation in web IR. In the next section we outline the specific recommendations gleaned from the literature reviewed above that were incorporated into the VSE.

Figure 1 The VSE interface (see online version for colours)

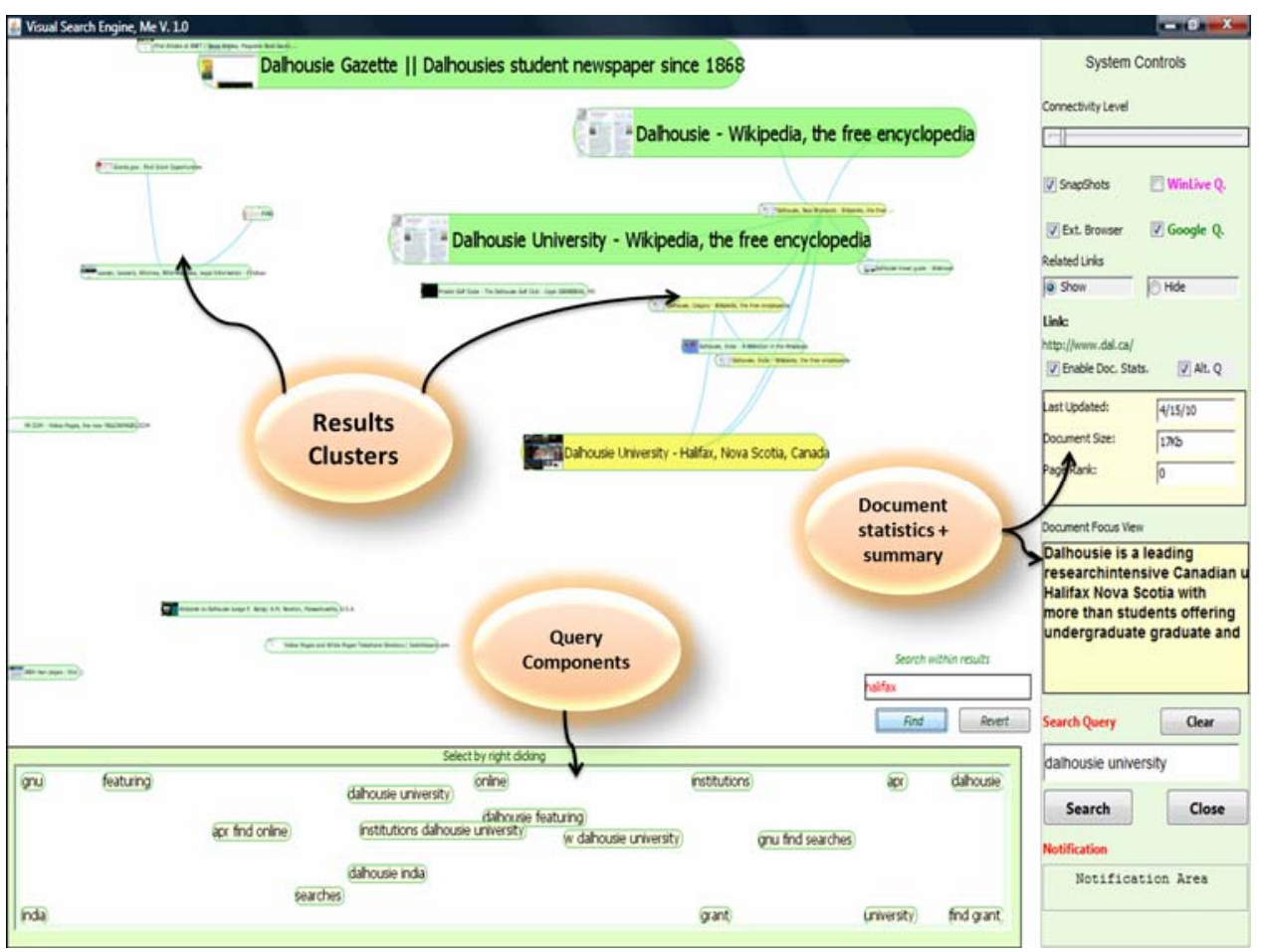

Source: Alhenshiri et al. (2010a) 


\section{Recommendations}

Web search often leads to very large result sets, with high degrees of feature dimensionality. High levels of recall with low precision are also frequently found. The search process usually discovers the results intended by the user, yet those results are incorrectly prioritised by the ranking process. Consequently, the role of visualisation in web search should take into consideration the following aspects and design goals:

- Visualisation should be applied on top of additional filtering and ranking mechanisms in order to improve the quality of the returned set. Additional filtering and re-ranking can be utilised for the purpose of minimising the effect of stale data and redundancy in web search results.

- Visualisation should provide customisable presentations to permit users to navigate through the returned matches. When allowed to customise, users can apply their own filtering of the returned results.

- The use of visualisation requires utilising more features of web documents such as page updates, page credibility, and page access rates to convey stronger insights about the result hits.

- Visualisation should consider the familiarity of users with text rendering. Reliance on sophisticated visual objects such as 3D metaphors may complicate the process of understanding the results.

- The design of visualisation techniques should consider the domain expertise of the intended user especially in the case of The Web. Most web search users are non-professionals (Laycock, 2005). They are casual users with no specific training.

\section{Conclusions and future work}

In this paper, several aspects of interactive use of visualisation in web IR were discussed. It is clear that exploiting interactive visualisation in both processes of query reformulation and results presentation in web search holds great promises. Users' ability to detect information scent in the rendered results is largely augmented through the use of visual rendering. Further research would consider the aspects discussed in Section 5 to improve the effectiveness of users searching The Web.

The VSE prototype search engine incorporated every recommendation gleaned from the literature we reviewed above. To make search engines that are suitable for collections as big as the WWW, systems like VSE will need to be more robust. Research is needed to determine the relative merits of visualisation features to enable developers to hone techniques to suit the users of tomorrow. We recommend that such research be aimed at identifying aspects of visualisation that allow users to gather information more effectively. Our research aims at building information gathering tools that would improve the accomplishment process for this type of tasks through the use of visualisation in addition to visual clustering.

Balancing the control between the search engine and the user may lead to more effective searches. Moreover, with the growth of The Web content, more aspects of visualisation should be employed in the process of web IR. For our part, we will also 
investigate the role of visualisation in the subtask of web information organisation as discussed by Alhenshiri et al. (2010b).

\section{References}

Abrams, A.R. and Law, B.M. (2002) 'Random visual noise impairs object-based attention', Journal of Experimental Brain Research, Vol. 142, No. 3, pp.349-353, SpringerLink.

Alhenshiri, A., Watters, C., Shepherd, M. and Brooks, S. (2010a) 'Improving the visual presentation of web search results', Proceedings of the 5th International Conference on Digital Information Management, Thunder Bay, ON, Canada.

Alhenshiri, A., Watters, C., Shepherd, M. and Duffy, J. (2010b) 'Web information gathering tasks: a framework and research agenda', Proceedings of the International Joint Conference on Knowledge Discovery, Knowledge Engineering and Knowledge Management, Valencia, Spain, to appear.

Alonso, O. and Baeza-Yates, R. (2003) 'Alternative implementation techniques for web text visualisation', Proceedings of 1st Latin American Web Congress, CA, USA, pp.202-204.

Bonnel, N., Lemaire, V., Cotarmanac'h, A. and Morin, A. (2006) 'Effective organization and visualisation of web search results', Proceedings of the 24th IASTED International Multi-Conference on Internet and Multimedia Systems and Applications, Innsbruck, Austria, pp.209-216.

Card, S.K., Mackinlay, J.D. and Shneiderman, B. (1999) Readings in Information Visualisation: Using Vision to Think, Morgan Kaufman Publishers, San Francisco, CA, USA.

Cellary, W., Wiza, W. and Walczak, K. (2004) 'Visualizing web search results in 3D', Computer Journal, Vol. 37, No. 5, pp.87-89.

Grewal, R.S., Jackson, M., Burden, P. and Wallis, J. (2000) 'Visual representation of search-engine queries and their results', Proceedings of the 1st International Conference on Web Information Systems Engineering, Hong Kong, China, Vol. 1, pp.352-356.

Havre, S., Hetzler, E., Perrine, K., Jurrus, E. and Miller, N. (2001) 'Interactive visualisation of multiple query results', Proceedings of the 2001 IEEE Symposium on Information Visualisation, San Diego, California, USA, pp.105-112.

Hoeber, O. and Yang, X.D. (2009) 'HotMap: supporting visual exploration of web search results', Journal of the American Society for Information Science and Technology, Vol. 60, No. 1, pp.90-110.

Joho, H. and Jose, J.M. (2006) 'A comparative study of the effectiveness of search result presentation on the web', SpringerLink Lecture Notes in Computer Science, Vol. 3936, pp.302-313.

Kawano, H. (2000) 'Overview of Mondou web search engine using text mining and information visualizing technologies', Proceedings of the International Conference on Digital Libraries, Kyoto, Japan, pp.234-244.

Kunz, C. and Botsch, V. (2002) 'Visual representation and contextualization of search results: list and matrix browser', Proceedings of the 2002 International Conference on Dublin Core Metadata Applications, Florence, Italy, pp.229-234.

Laycock, J. (2005) 'Most search engine users still native', Search Engine Guide, available at $\mathrm{http} / /$ www.searchengineguide.com/jennifer-laycock/most-search-engine-users-still-naive.php (accessed on 19 February 2009).

Manning, C.D., Raghavan, P. and Schütze, H. (2008) Introduction to Information Retrieval, Cambridge University Press.

Marchionini, G. (1997) Information Seeking in Electronic Environments, Cambridge University Press.

Miller, G.A. (1990) 'WordNet: an on-line lexical database', International Journal of Lexicography, Vol. 3, No. 4, pp.285-303. 
Mukherjea, S. and Hara, Y. (1999) 'Visualizing world-wide web search engine results', Proceedings of the IEEE International Conference on Information Visualisation, London, UK, pp.400-405.

Roberts, J.C., Boukhelifa, N. and Rodgers, P. (2004) 'Visual depictions of search results: using glyphs and coordinated multiple-views', YLEM Journal, Vol. 24, No. 2, pp.8-10.

Shidara, M. and Richmond, J.R. (2005) 'Effect of visual noise on pattern recognition', Journal of Experimental Brain Research, Vol. 163, No. 2, pp.239-241, SpringerLink.

Solis-Oba, R. and Alhenshiri, A. (2007) 'Improving results for short web queries using preserved query knowledge', Proceedings of the 1st International Conference on Digital Communications and Computer Applications, Amman, Jordan, pp.634-649.

Spink, A., Wolfram, D., Jansen, M. and Saracevic, T. (2001). 'Searching the web: the public and their queries', Journal of the American Society for Information Science and Technology, Vol. 52, No. 3, pp.226-234.

Suvanaphen, E. and Roberts, J.C. (2004) 'Textual difference visualisation of multiple search results utilizing detail in context', Proceedings of the Theory and Practice of Computer Graphics Conference, Bournemouth, UK, pp.2-8.

Teevan, J., Cutrell, E., Fisher, D., Drucker, S.M., Ramos, G., André, P. and Hu, C. (2009) 'Visual snippets: summarizing web pages for search and revisitation', Proceedings of the 27th International Conference on Human Factors in Computing Systems, Boston, MA, pp.2023-2032.

Vincente, K.J. and Rasmussen, J. (1990) 'The ecology of human machine systems: mediating direct perception in complex domain', Journal of Ecological Psychology, Vol. 2, No. 3, p.207.

Zaveskey, E., Chang, S. and Yang, C. (2008) 'Visual Islands: intuitive browsing of visual search results', Proceedings of the 2008 International Conference on Content-based Image and Video Retrieval, Niagara Falls, Canada, pp.617-626. 